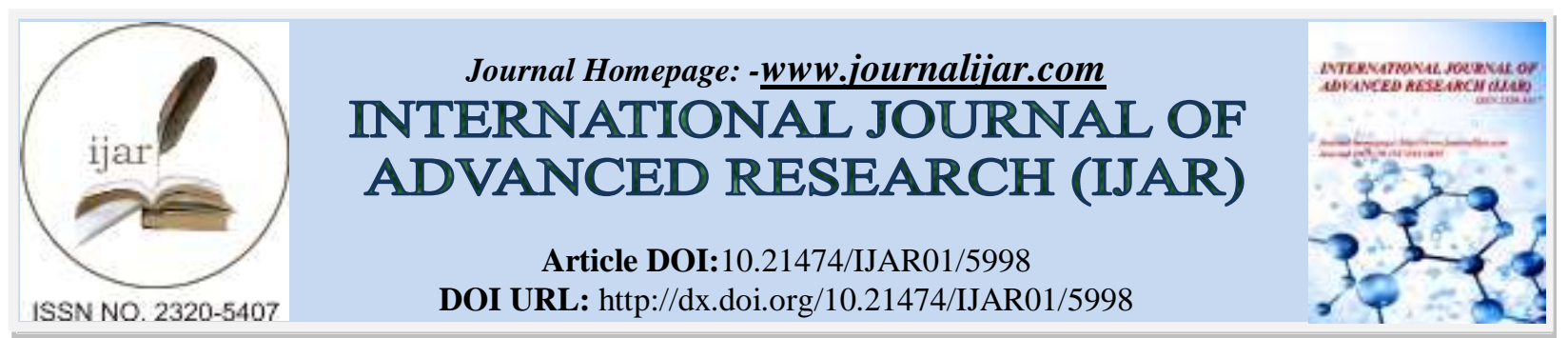

RESEARCH ARTICLE

\title{
IN SILICO DOCKING STUDIES OF ANTI-DIABETIC AND BREAST CANCER ACTIVITY BY N'-(1-BENZYLPIPERIDIN-4-YLIDENE)-2-CYANOACETOHYDRAZIDE.
}

\author{
*K. Tharini and K. Sundaresan. \\ Department of chemistry, Govt Arts College, Trichy-22, TamilNadu, India.
}

\section{Manuscript Info}

Manuscript History

Received: 07 October 2017

Final Accepted: 09 November 2017

Published: December 2017

\section{Keywords:-}

insilico docking, CDOCKER.

\begin{abstract}
The molecular docking approach can be used to model the interaction between a small molecule and a protein at the atomic level, which allow us to characterize the behavior of small molecules in the binding site of target proteins as well as to elucidate fundamental biochemical processes. In our present study, to predict the activity of our synthesized compounds N'-(1-benzylpiperidin-4-ylidene)-2cyanoacetohydrazide using in silico approaches by molecular docking. In silico molecular docking studies were carried out using BIOVIA Discovery Studio (DS) 2017 software. It has been found that the molecule has superior and strong binding affinity in the both proteins (2YAT and 1IR3) compare with standard drug. Possible binding modes between the ligands and this target proteins were studied by CDOCKER (CHARMm-based DOCKER) protocol incorporated within DS. Molecular docking was utilized to prove that similar compounds can bind to receptor protein treatment of breast cancer using in silico approach.
\end{abstract}

Copy Right, IJAR, 2017,. All rights reserved.

\section{Introduction:-}

Breast cancer is a serious health concern in India causing the highest mortality rate in females, which occurs due to uncontrolled cell division and can be metastasize to other parts of the human body. The available drugs and treatments are not satisfactory as they do not completely eradicate the cancerous cells from the body. Currently.Tamoxifen used as a standard drug for breast cancer. Tamoxifen is a nonsteroidal triphenyl ethylene derivative that binds to the estrogen receptor. It has both estrogenic and antiestrogenic actions, depending on the target tissue. It is strongly antiestrogenic on mammary epithelium, hence its use in both the prevention and treatment of breast cancer; it is proestrogenic on uterine epithelium, hence the current controversy regarding its safety in cancer prevention especially since an increased incidence of endometrial carcinoma has been found in women treated chronically with tamoxifen. It is therefore inappropriate to refer to tamoxifen simply as an antiestrogen. The term selective estrogen receptor modulator is more appropriate. The mechanism of action of tamoxifen is complex. Clearly, its principal mechanism of action is mediated by its binding to the estrogen receptor and the blocking of the proliferative actions of estrogen on mammary epithelium. One suggested mechanism for this antiproliferative action is the induction by tamoxifen of the synthesis of the cytokine transforming growth factor- $\beta$ (TGF- $\beta$ ), which acts as a negative autocrine regulatory N'-(1-benzylpiperidin-4-ylidene)-2-cyanoacetohydrazide. 
Diabetes mellitus is a most common disorder of endocrine gland which is caused due to deficiency in insulin production or ineffectiveness of insulin produced. So this a deficiency of insulin result in improper metabolism of glucose which have harmful effect in the body system, in particular the blood vessels and nerves. Diabetes affects more than 171 million people worldwide and according to the resent study, this population may be increased 366 million by 2030. Glibenclamide was the standard drug used to stimulate insulin from beta cells of islets of langerhans many years in research. So, Glibenclamide used as a standard compound in this study.

The side effects of the currently used drug made us to explore an alternative and synthesis approach to finding out new drug compound which are having anti-diabetes and anti-breast cancer activity and also not having any side effects to human normal cell.

In this study, to predict the activity of our synthesized compounds N'-(1-benzylpiperidin-4-ylidene)-2cyanoacetohydrazide using in silico approaches by molecular docking. Molecular docking was utilized to prove that similar compounds can bind to receptor protein treatment of breast cancer using in silico approach.

\section{Materials and methods:-}

\section{Synthesis of N'-(1-benzylpiperidin-4-ylidene)-2-cyanoacetohydrazide}

A mixture of N-benzyl pipperidone $(0.1 \mathrm{~mol})$, cyanoacetichydrazide $(0.1 \mathrm{~mol})$ in the presence of few drops of concentrated acetic acid in methanol was refluxed for 2 hours. After the completion of reaction, the reaction mixture was cooled to room temperature. The solid product was separated by filtration and washed with warm water and recrystallized by methanol to afford N'-(1-benzylpiperidin-4-ylidene)-2-cyanoacetohydrazide.

In our present study, in silico molecular docking studies were carried out using BIOVIA Discovery Studio (DS) 2017 software.

\section{Preparation of protein:-}

The X-ray crystal structure of human breast cancer protein 2YAT and protein insulin receptor 1IR3 for in this antidiabetes mellitus study were retrieved from RCSB Protein Data Bank (http:// www.rcsb.org/pdb). Hydrogen's were added to the protein 2YAT and 1IR3 by applied the forcefield, then energy minimized using CHARM forcefield in DS.

\section{Ligand preparation:-}

The standard drug N'-(1-benzylpiperidin-4-ylidene)-2-cyanoacetohydrazide Tamoxifen, Glibenclamide and N'-(1benzylpiperidin-4-ylidene)-2-cyanoacetohydrazide were drawn in chemdraw software, subsequently energy minimized and saved in SDF file format for docking studies.

\section{Result and Discussion \\ Docking studies:-}

Computational ligand-target docking approach was used to analyze structural complexes of the 2YAT and 1IR3 with Tamoxifen, Glibenclamide and N'-(1-benzylpiperidin-4-ylidene)-2-cyanoacetohydrazide (ligands) in order to understand the structural basis of this target proteins. Possible binding modes between the ligands and this target proteins were studied by CDOCKER (CHARMm-based DOCKER) protocol incorporated within DS. The algorithm offers full ligand flexibly and employs CHARMm force fields. Ligand binding affinity was calculated using CDOCKER energy, CDOCKER Interaction energy, Hydrogen bonds, binding energies, protein energy and ligand protein complex energy. The CDOCKER energy mentioned in negative values. More negative value energy indicated as higher binding affinity of the ligands with target protein.

\section{Anti-cancer activity:-}

Human estrogen receptor (2YAT) has been exploited as a main therapeutic target for breast cancer. The X-ray crystallography of this protein was $2.60(\AA)$ and it contain 252 amino acids (Figure 1 ).

In this study, the N'-(1-benzylpiperidin-4-ylidene)-2-cyanoacetohydrazide forms non-bonding interaction with active site of human estrogen receptor protein. This N'-(1-benzylpiperidin-4-ylidene)-2-cyanoacetohydrazide forms van der Waals interactions with amino acids Leu (346, 428, 384, 387, 525, 391), Met (388, 528), Phe 404, Ala 350, Gly 521 and Ile 424 in active site. The residue Glu 423 forms electrostatic interaction with synthesis N'-(1benzylpiperidin-4-ylidene)-2-cyanoacetohydrazide (Figure2A and B) The CDOCKER energy of N'-(1- 
benzylpiperidin-4-ylidene)-2-cyanoacetohydrazide in this protein is $-27.8982 \mathrm{Kcal} / \mathrm{Mole}^{-1}$. CDOCKER energy is a total calculation of electrostatic and van der Waals interaction of the N'-(1-benzylpiperidin-4-ylidene)-2cyanoacetohydrazide in 2YAT. The two aromatic ring in the N'-(1-benzylpiperidin-4-ylidene)-2cyanoacetohydrazide forms more van der Waals interactions with hydrophobic residues.

Similarly, the standard drug Tamoxifen shown $-7.62965 \mathrm{Kcal} / \mathrm{mol}^{-1}$ CDOCKER energy. It forms van der Waals and electrostatic interactions with active site of 2YAT protein (Figure 3A and B). Additionally, one pi-pi bond involved between the Tamoxifen and the receptor. However, the CDOCKER energy of the standard breast cancer drug Tamoxifen $\left(-7.62965 \mathrm{Kcal} / \mathrm{mol}^{-1}\right)$ shown lowest binding affinity compare with our synthesis $\mathrm{N}^{\prime}$-(1-benzylpiperidin4-ylidene)-2-cyanoacetohydrazide (-27.8982 Kcal/Mole $\left.{ }^{-1}\right)$. It revealed that, the N'-(1-benzylpiperidin-4-ylidene)-2cyanoacetohydrazide exposed good binding affinity than Tamoxifen in the breast cancer protein.

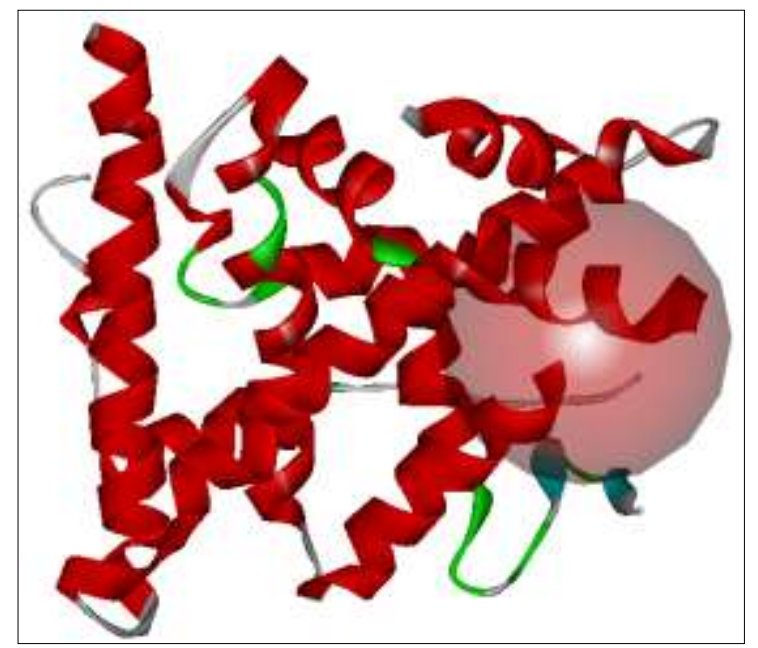

Figure 1:-The X-ray crystallography of human estrogen receptor (2YAT) protein at resolution $2.60 \AA$ with active

Figure $2(\mathrm{~A})$ site sphere.

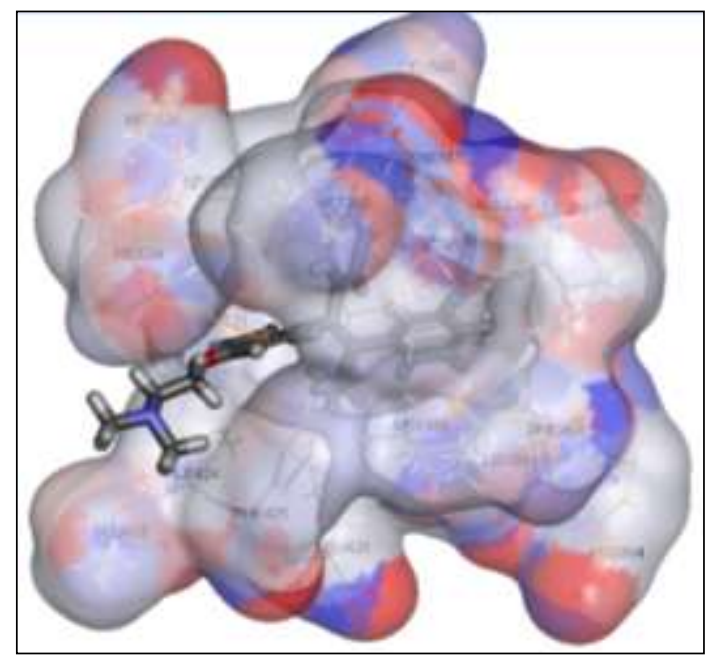


Figure 2 (B)

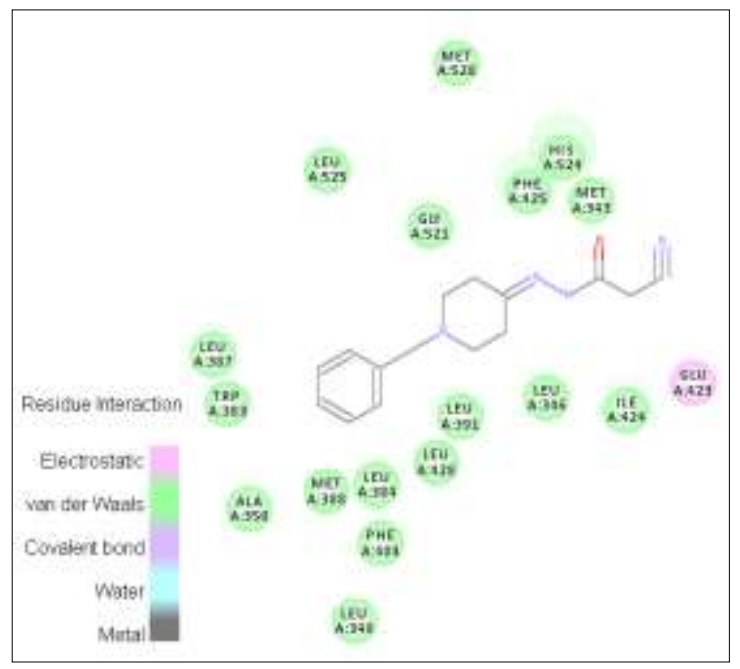

Figure 2(A) 3D and 2(B) (2D) interaction of the N'-(1-benzylpiperidin-4-ylidene)-2-cyanoacetohydrazide in 2YAT Figure 3: (A) active site protein

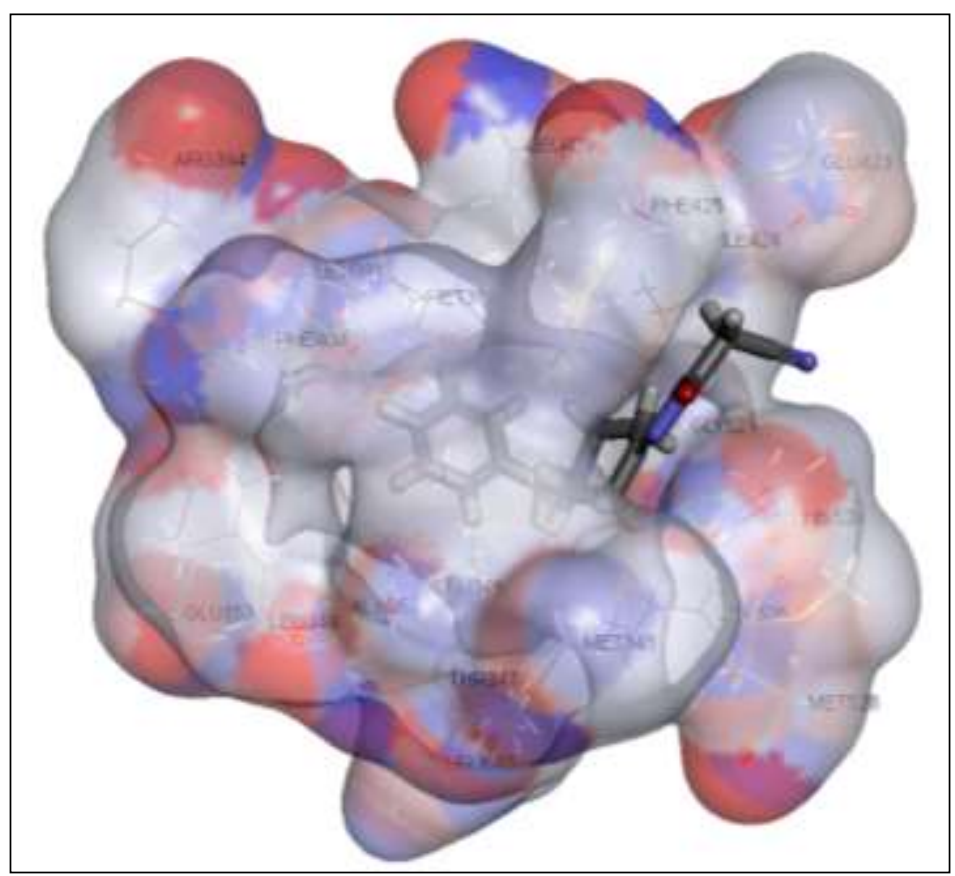


Figure 3 (B)

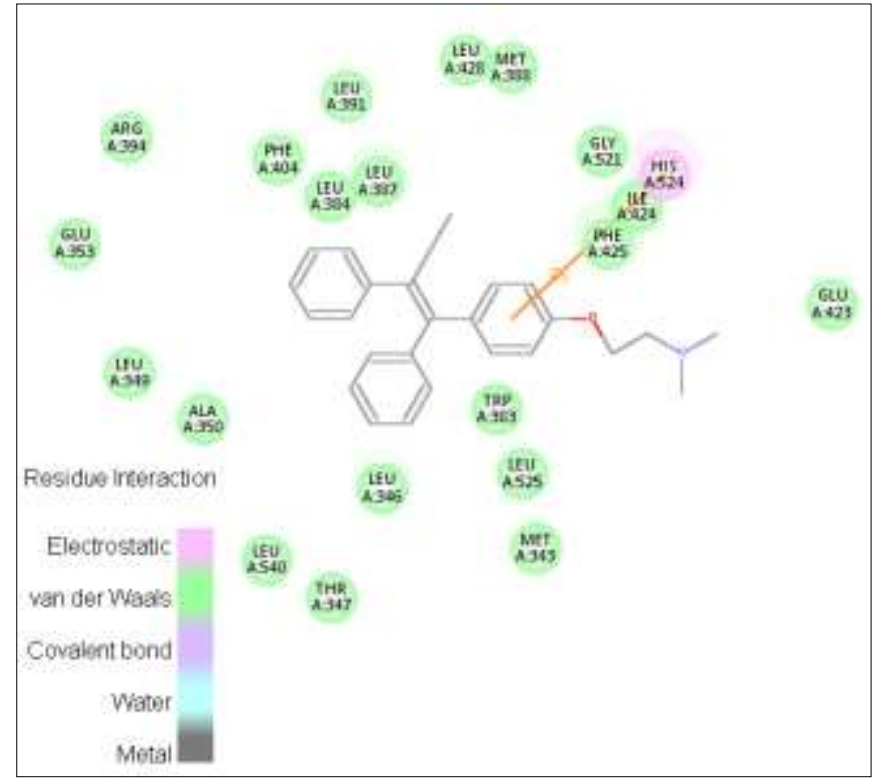

Figure 3 (A) 3D and (B) 2D interaction of the Tamoxifen in 2YAT active site protein

\section{Anti-diabetic activity:-}

In this study, the ligand poses were analyzed and interaction of ligand N'-(1-benzylpiperidin-4-ylidene)-2cyanoacetohydrazide with the 1IR3 protein structure was studied on the basis of $\mathrm{H}$-bonding made by the poses to the receptor N'-(1-benzylpiperidin-4-ylidene)-2-cyanoacetohydrazide and close contacts (Vander Waals clashes) between receptor and N'-(1-benzylpiperidin-4-ylidene)-2-cyanoacetohydrazide. As it is well known, $\mathrm{H}$ bonds play an important role for the structure and function of biological N'-(1-benzylpiperidin-4-ylidene)-2cyanoacetohydrazide, especially for the enzyme catalysis.

The docking study was performed between insulin receptor (1IR3) and N'-(1-benzylpiperidin-4-ylidene)-2cyanoacetohydrazide as well as standard anti-diabetic drug Glibenclamide. The CDOCKER energy value is 35.5075 and $32.4341 \mathrm{Kcal} / \mathrm{mol}^{-1}$ for N'-(1-benzylpiperidin-4-ylidene)-2-cyanoacetohydrazide and Glibenclamide respectively. The 3D and 2d interactions of the both ligands are depicted in Figure 4A, 4B and 5A, 5B. Interestingly, the CDOCKER value of the N'-(1-benzylpiperidin-4-ylidene)-2-cyanoacetohydrazide is higher than the standard anti-diabetic drug. The N'-(1-benzylpiperidin-4-ylidene)-2-cyanoacetohydrazide form one hydrogen bond with Asp1150 and two hydrogen bond with Lys1030 residues. However, the Glibenclamidedrug form only one hydrogen bond with Lys1030 and one Pi-Pi interaction with Arg1136. It revealed that the N'-(1-benzylpiperidin-4-ylidene)-2cyanoacetohydrazide has strong binding affinity than Glibenclamide drug.

Figure 4 (A)

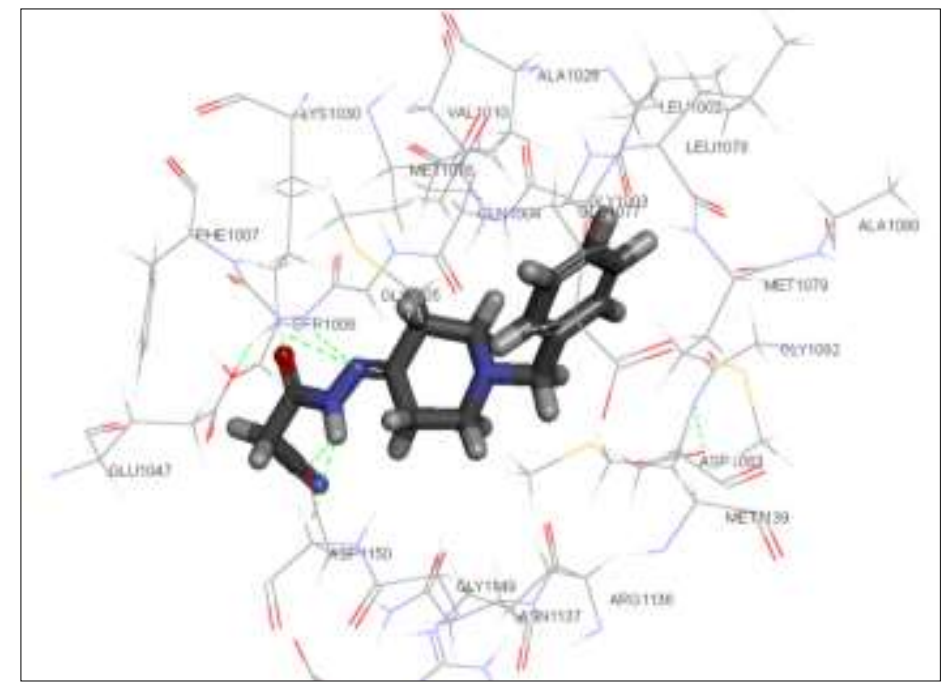


Figure 4 (B)

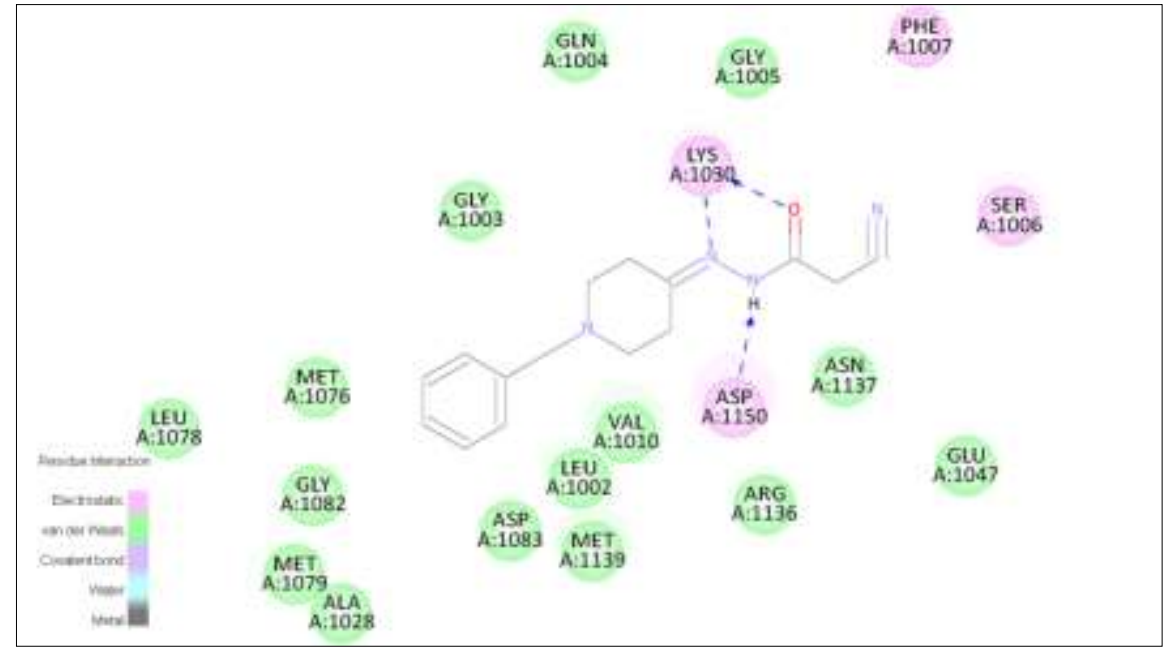

Figure 4(A) 3D and (B) (2D) interaction of the $\mathrm{N}$ '-(1-benzylpiperidin-4-ylidene)-2-cyanoacetohydrazide in 2YAT Figure 5 (A) active site protein

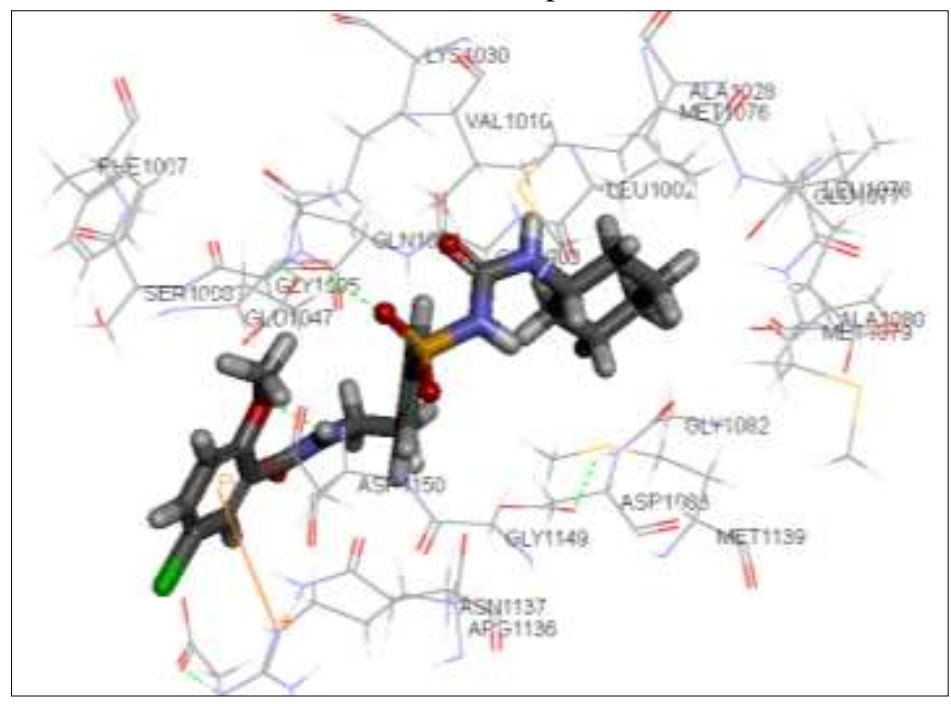

Figure 5( B)

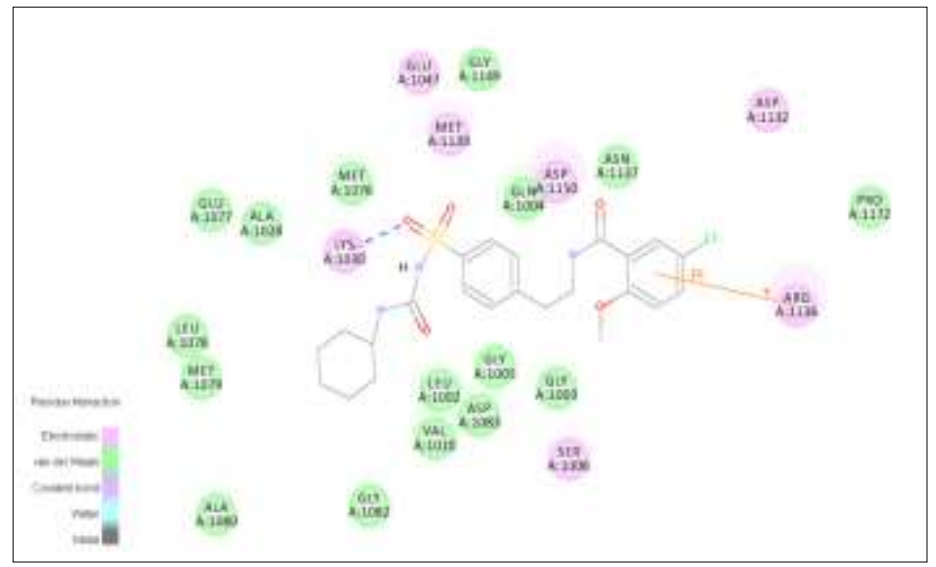

Figure 5 A) 3D and (B) 2D interaction of the Glibenclamide in 2YAT active site protein 


\section{Conclusion:-}

The aim of the present study was to identify the anti-diabetic and breast cancer activity of the N'-(1-benzylpiperidin4-ylidene)-2-cyanoacetohydrazide molecule. We have successfully achieved the docking of N'-(1-benzylpiperidin-4ylidene)-2-cyanoacetohydrazide in the target protein of anti-diabetic and breast cancer. This molecule has superior and strong binding affinity in the both proteins (2YAT and 1IR3) compare with standard drug. Finally, we concluded that the molecule N'-(1-benzylpiperidin-4-ylidene)-2-cyanoacetohydrazide has potent anti-diabetic and breast cancer effect which could be used as for the management of cancer and diabetes effectively.

\section{References:-}

1. Aunuradha Sharma, Vaibhav Walia, Monika Gahlawat "Molecular Docking Studies of N-(2-Benzoylphenyl)-LTyrosine Derivatives with Anti-Diabetic Activity of Type 2 Diabets" PharmatutorMagazine Vol.3,Issue 2. P 58-68.

2. TejasviKhamkar, MayureshAbhyankar, Gauri Tendulkar, VenkatesanGopieshKhanna, Krishnan Kannabiran "In SilicoMoleculr Docking of Marine Drugs Against Cancer Proteins" Advancec in Chemical Science Vol.2 Issue.2, June 2013.

3. Anuradha Sharma, VaibhavWalia, Gahlawat “ Molecular Docking Studies of N-(2-Benzoylphenyl)-L-Tyrosine Derivatives with Anti-Diabetic Activity of Type 2 Diabets" PharmatutorE-ISSN: 2347-7881 P 58-68.

4. JayasreeGanugapati, and Sruthiswarna "Molecular Docking Studies of Anti-diabetic Activity of Chinnamon Compounds" Asian Journal of Pharmaceutical and Clinical ResearchVol 7, Suppl 2,2014

5. A.Bharathi, SelvarajMohanaRoopan, C.S.Vasavi, G.A. Gayathri and M.Gayathri“" InSilico Molecular Docking and In Vitro Antidiabetic Studies of Dihydropyrimido[4,5-a] acridin -2-amines" BiomedResearch International Vol 2014,Article ID 971569.

6. Danish Ahmed et al "Molecular docking analysis and antidiabetic activity of Rifabutin against STZ-NA induced diabetes in albino wistar rats" Journal of Basic and Applied Sciences Apr 2017.

7. R.Maheswari, J.Manjula "Synthesis, Characterization and Biological Applications of Benzohydrazide derivatives" International Journal of Applied Research 2015;1(10): P 587-592. 